4

\title{
Radio Pulsars
}




\title{
PLANETS AROUND PULSARS
}

\author{
A. WOLSZCZAN \\ Penn State University, Dept. of Astronomy and Astrophysics \\ 525 Davey Laboratory, University Park, PA 16802, U.S.A.
}

\begin{abstract}
A discovery of three terrestrial-mass planets orbiting the millisecond pulsar PSR B1257+12 and a subsequent detection of the predicted effect of gravitational interaction between the two more massive planets confirms that the first extrasolar planetary system has been identified and that pulsars can be successfully used as probes of planetary dynamics. In the absence of detections of planet-sized objects around Sun-like stars, planets orbiting a precise pulsar clock represent a unique source of information concerning the origin and evolution of planetary systems.
\end{abstract}

\section{Introduction}

A detection of planetary companions to stars other than the Sun has been one of the most challenging tasks of modern observational astrophysics. Either a positive or negative outcome of searches for extrasolar planets would directly address fundamental problems associated with the origin of the Solar System and it would be instrumental in the process of understanding the relation of Earth and terrestrial life to the rest of the universe. So far, no direct detection of planets orbiting a Sun-like star has been reported, in spite of a growing number of tantalizing hints (Sargent \& Beckwith 1993). Instead, a discovery of planets around a rapidly rotating, old neutron star, the 6.2-millisecond radio pulsar PSR B1257+12, has been announced in early 1992 (Wolszczan \& Frail 1992).

This paper describes the detection of the PSR B1257+12 planets, their confirmation through a successful measurement of the planetary perturbations and the most important consequences of this discovery. 


\section{Planets Around PSR B1257+12}

A 6.2-millisecond pulsar, PSR B1257+12, was discovered in 1990 during a pulsar search conducted with the 305-m Arecibo radio telescope (Wolszczan 1991). The analysis of the follow-up timing observations has revealed large, quasi-periodic deviations of the times-of-arrival (TOAs) of pulses predicted by the standard timing model from the actually observed TOAs. Further examination of the post-fit residuals has shown that they can be decomposed into two steady, almost sinusoidal oscillations with the periods of 66.6 and 98.2 days (Figs. 1 b,c).

A number of possible sources of this uncommon timing behavior of PSR B1257+12 have been considered. These included a timing noise caused by the seismic activity of a rapidly rotating neutron star, propagation effects in the circumpulsar medium, neutron star precession, errors in timing analysis and a variety of possibilities of instrumental origin. All these alternatives have been successively eliminated leaving a Keplerian orbital motion of two planet-mass objects around the pulsar as the most plausible explanation of the periodicities in the pulsar's timing residuals. More detailed analysis of the TOA measurements has led to a detection of an additional, much lower amplitude periodicity which was interpreted as a signature of the presence of yet another, very low-mass object, in a 25.3-day orbit around the pulsar (Fig. 1a). The resulting 3-planet timing model and the basic characteristics of the PSR B1257+12 planetary system are shown in Table 1 with the planets labeled $A, B$ and $C$ in order of increasing distance from the pulsar (see Wolszczan (1994) and references therein for a detailed description of the development of the timing model for PSR B1257+12).

\section{Detection of Planetary Perturbations}

The question of detectability of gravitational perturbations of the orbits of the pulsar planets has been raised soon after the announcement of the PSR B1257+12 system (Rasio et al. 1992; Malhotra et al. 1992). It has been pointed out that an approximately 3:2 ratio of the orbital periods of the two larger planets creates a near-resonance condition which leads to accurately predictable and possibly measurable periodic perturbations of the two orbits. A detection of planetary perturbations has been commonly regarded as the most unambiguous and perhaps the only way to produce a final proof that the timing behavior of PSR B1257+12 is due to orbital dynamics involving at least two planet-sized bodies.

Detailed analyses of the dynamics of the PSR B1257+12 planetary system and their effects on pulsar timing have been carried out by Malhotra (1993), Rasio et al. (1993) and Peale (1993). The observable consequences of a mutual gravitational interaction between the two planets include near- 

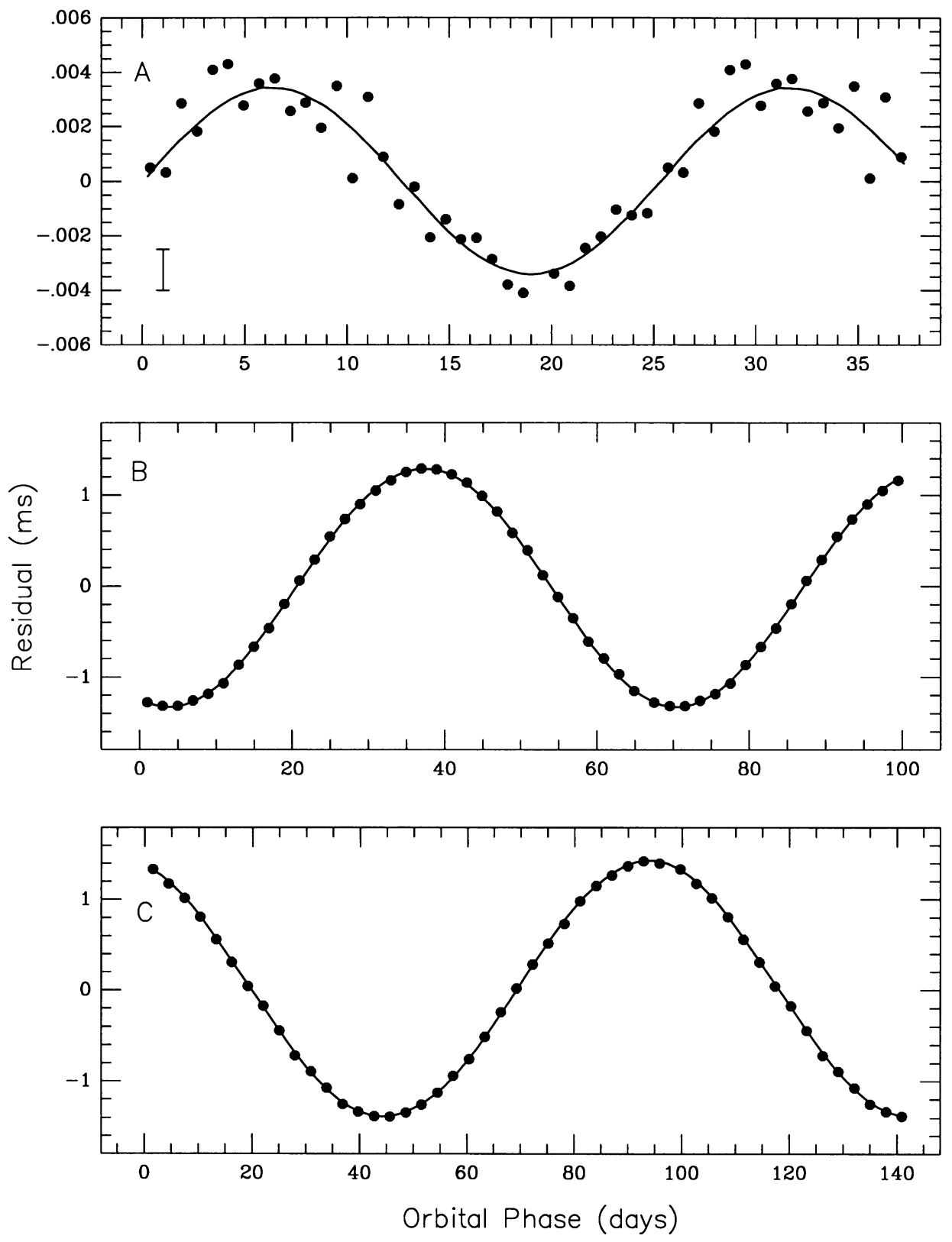

Figure 1. The post-fit residuals of pulse arrival times from PSR B1257+12 folded modulo the orbital periods of 25.34 days (planet $A$ ), 66.54 days (planet $B$ ) and 98.22 days (planet $C)$, over a 3-year span of timing observations. In each case, the arrival time variations due to the other two planets have been fitted out. For planet $A$, a $2 \sigma$ uncertainty in residuals is indicated by the error bar. For planets $B$ and $C$, the uncertainties are too small to be shown. 


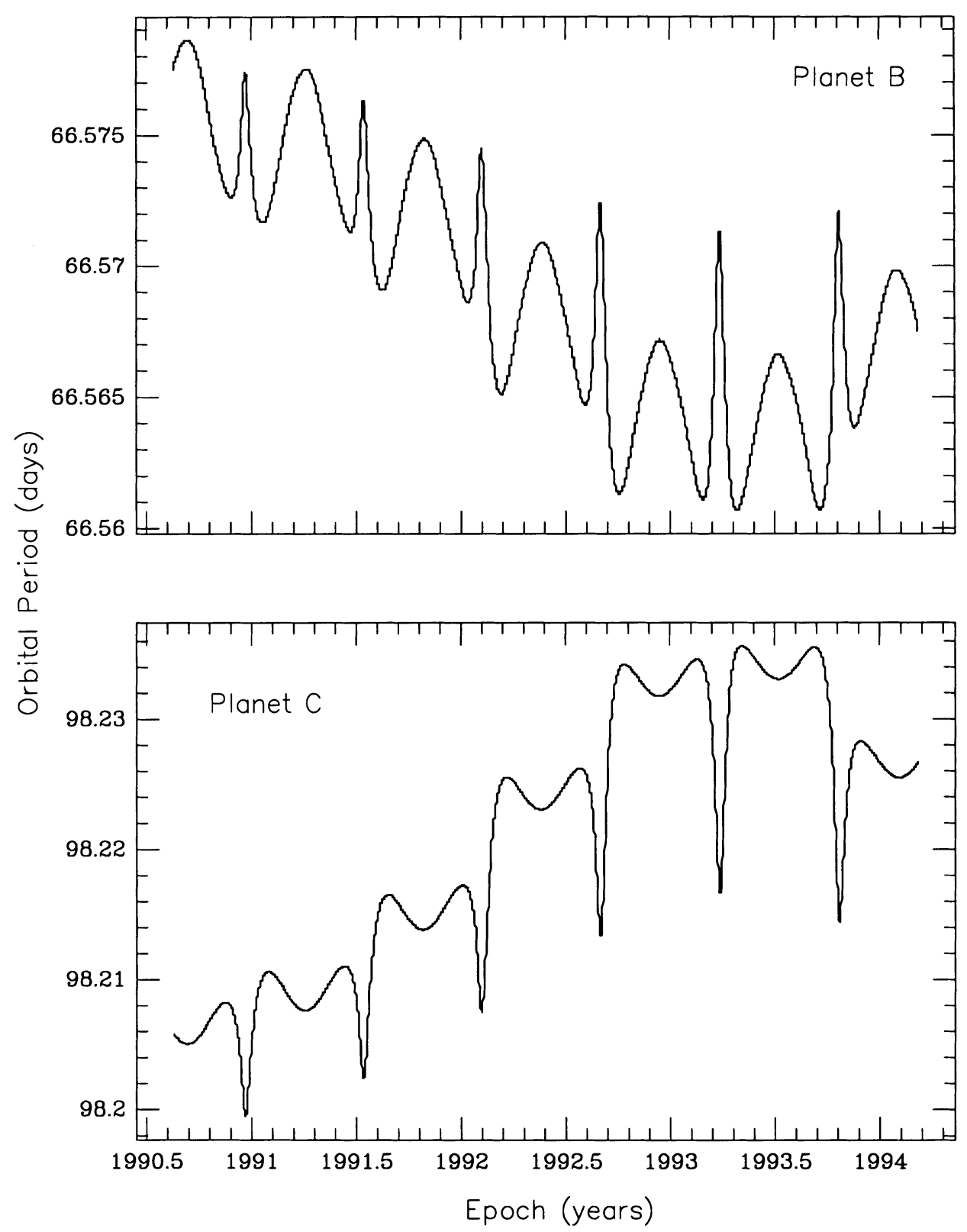

Figure 2. The predicted perturbations of orbital periods derived from numerical integration of equations of motion of planets $B$ and $C$. 
TABLE 1. Parameters of the PSR B1257+12 planetary system

Pulsar parameters

Rotational period

Period derivative

Second period derivative

Right ascension, $\alpha_{1950}$

Declination, $\delta_{1950}$

Proper motion in $\alpha$

Proper motion in $\delta$

Epoch

Dispersion measure

$0.0062185319388187(2) \mathrm{s}$
$1.14334(6) 10^{-19} \mathrm{~s} \mathrm{~s}^{-1}$
$4.5(9) 10^{-30} \mathrm{~s}^{-1}$
$12^{h} 57^{m} 33^{s} .12730(3)$
$12^{\circ} 57^{\prime} 06^{\prime \prime} .406(1)$
$46.4(6) \mathrm{mas} \mathrm{yr}^{-1}$
$-82.9(9) \operatorname{mas~yr}^{-1}$
$\mathrm{JD} 2448788.9$
$10.186(1) \mathrm{pc} \mathrm{cm}^{-3}$

Keplerian orbital parameters
$B$

$1.3106(6)$
$0.0182(9)$
$2448770.3(6)$
$5748713(90)$
$249(3)$

$C$

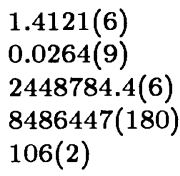

Parameters of the planetary system

$\begin{array}{llll}\text { Planet mass }\left(M_{\oplus}\right) & 0.015 / \sin \mathrm{i}_{1} & 3.4 / \sin \mathrm{i}_{2} & 2.8 / \sin \mathrm{i}_{3} \\ \text { Distance from the pulsar (AU) } & 0.19 & 0.36 & 0.47 \\ \text { Orbital period (days) } & 25.34 & 66.54 & 98.22\end{array}$

resonant, periodic variations of the elements of their orbits and the superimposed short-term, non-resonant fluctuations (Fig. 2). If the planetary masses are not too big, the relevant period is simply given in terms of the mean angular velocities of the planets, $n_{1}$ and $n_{2}$, as $2 \pi /\left(2 n_{1}-3 n_{2}\right)=5.56$ years and the predicted maximum amplitude of the corresponding timing residuals (after fitting out the two non-interacting orbits) is a function of planetary masses and the total time span of observations (Peale 1993).

In timing observations, the perturbations should manifest themselves in the form of oscillating residuals, if the least-squares fit of a timing model to data assumes fixed-parameter, non-interacting orbits. These oscillations are characterized by time scales of the order of orbital periods of the planets and by amplitudes which depend on the planet-to-pulsar mass ratios. Using the timing model of Table 1 , and with the planetary masses, $m_{1,2}$ expressed in terms of the Earth mass and $M_{\mathrm{psr}}$ in units of $1.4 \mathrm{M}_{\odot}$, these mass ratios are given by:

$$
\frac{m_{1}}{M_{\mathrm{psr}}}=\frac{3.4}{\sin i_{1} M_{\mathrm{psr}}^{1 / 3}}
$$




$$
\frac{m_{2}}{M_{\mathrm{psr}}}=\frac{2.8}{\sin i_{2} M_{\mathrm{psr}}^{1 / 3}}
$$

for planets $B, C$ and orbital inclinations $i_{1}, i_{2}$, respectively. Since the effect of a mutual inclination of the two orbital planes would have to be quite large to become detectable (Malhotra 1993), it is reasonable to assume coplanar orbits. Consequently, with $i_{1}=i_{2}=i$, the perturbation amplitude becomes a function of one variable, $(\sin i)^{-1} M_{\mathrm{psr}}^{-1 / 3}$, which is the only parameter governing the effect of planetary perturbations on pulse arrival times.

The result of a search for the best-fit perturbation model using the downhill simplex algorithm (Nelder \& Mead 1965) is shown in Fig. 3a. Compared to a timing model without planetary perturbations (Table 1), inclusion of this effect in the modelling process reduces the value of $\chi^{2}$ for the global fit by nearly $3 \%$, which is about 50 times the formal accuracy of the chosen minimization procedure. The presence of a well-defined minimum in the $\chi^{2}=\chi^{2}\left[(\sin i)^{-1} M_{\mathrm{psr}}^{-1 / 3}\right]$ curve confirms that planetary perturbations influence the observed pulse arrival times in the manner predicted by theory. The detection of this effect represents a proof that the pulse arrival time variations observed in PSR B1257+12 are due to orbital motion of planet-mass bodies with the dynamical characteristics that are not unlike those of the inner planets of the Solar System. This discovery is the first of a planetary system around a star other than the Sun. A more detailed description of the above analysis can be found in Wolszczan (1994).

Although the planetary perturbation modelling does not allow determination of all system parameters in a unique way, useful constraints on the masses of the pulsar and its planetary companions can be established in a straightforward manner. With the aid of Eqs. (1) and (2), the result of the perturbation model fitting (Fig. 3a) can be presented in the form of a pulsar mass-normalized planetary mass diagram (Fig. 3b). The uncertainty of the determination of a $\chi^{2}$ minimum in Fig. 3a defines a range of allowable masses in this diagram. It is further constrained by the highest possible orbital inclination $\left(i=90^{\circ}\right)$ and by the maximum neutron star mass (taken to be $2 \mathrm{M}_{\odot}$ ). Within these limits, the minimum pulsar mass is $\sim 1.2 \mathrm{M}_{\odot}$ and the range of possible pulsar masses for orbital inclinations close to $i=90^{\circ}$ includes both the typical theoretical value $\left(\sim 1.3 \mathrm{M}_{\odot}\right)$ (Woosley 1987$)$ and the observed average neutron star mass $\left(1.35 \pm 0.27 \mathrm{M}_{\odot}\right)$ (Thorsett et al. 1993). Furthermore, the masses of planets $B$ and $C$ must be similar to their respective "canonical", Earth-like values of $3.4 \mathrm{M}_{\oplus}$ and $2.8 \mathrm{M}_{\oplus}$ (Table 1 and Eqs. 1, 2) and the orbital inclinations are unlikely to be less than $60^{\circ}$ for any reasonable choice of a neutron star mass. No additional information concerning the orbit of planet $A$ can be extracted from this analysis, because its effect on the mutual perturbations of planets $B$ and $C$ is entirely negligi- 
ble. However, if the orbits of all three planets are approximately coplanar, planet $A$ must be a very low-mass, Moon-like object (Table 1 ).

\section{Pulsar Planets and Other Planetary Systems}

The observed characteristics of the planets and PSR B1257+12 itself, when confronted with current ideas concerning planetary formation (Levy 1993, Ruden 1993) and the origin and evolution of millisecond pulsars (Bhattacharya \& Van den Heuvel 1991), indicate that the planets are likely to have evolved in a circumpulsar disk of matter created from the remains of the pulsar's binary stellar companion. Therefore, most of the scenarios of the millisecond pulsar planet formation concern themselves with possible ways to transform a fraction of the companion's mass into a protoplanetary disk, implying that the planets would subsequently form in a manner similar to that envisioned for the origin of the Solar System (Podsiadlowski 1993; Phinney \& Hansen 1993). This indicates an interesting possibility that the planets around solar-type stars and pulsars may differ in their physical and chemical characteristics, but the fundamental features of the dynamics of their parent planetary systems should be comparable.

Pulsars accompanied by planets can be used as highly accurate probes of planetary dynamics. In the case of PSR B1257+12, a positive identification of planetary perturbations and the detection of a Moon-mass planet $A$ involved measurements and analysis of pulse arrival times at a microsecond precision level, which is equivalent to radial-velocity resolution of the order of $1 \mathrm{~mm} \mathrm{~s}^{-1}$ (a factor of $10^{4}$ better than the typical $10 \mathrm{~m} \mathrm{~s}^{-1}$ resolution achieved in modern single-line Doppler spectroscopy). These two techniques are compared in Fig. 4, in which the minimum detectable amplitudes of timing residuals, $\delta t$ and radial velocities, $\delta V_{\mathrm{r}}$ are expressed in terms of masses and orbital radii as:

$$
\begin{aligned}
\delta t & =c^{-1} \frac{m}{M} a \\
\delta V_{\mathrm{r}} & =G^{1 / 2} \frac{m}{(M a)^{1 / 2}}
\end{aligned}
$$

where $m$ is the planetary mass, $M$ is the mass of the central body, $a$ is the semi-major axis of the orbit and circular, "edge-on" orbits are assumed. Evidently, detections and dynamical studies of terrestrial-mass planets will remain beyond the reach of optical astrometry and Doppler spectroscopy in the foreseeable future, but they will be quite feasible with the pulse timing method.

The detection of pulsar planets dramatically emphasizes the value of enriching the strategies of planetary searches with non-standard approaches. At present, among more than twenty known millisecond pulsars, there are 

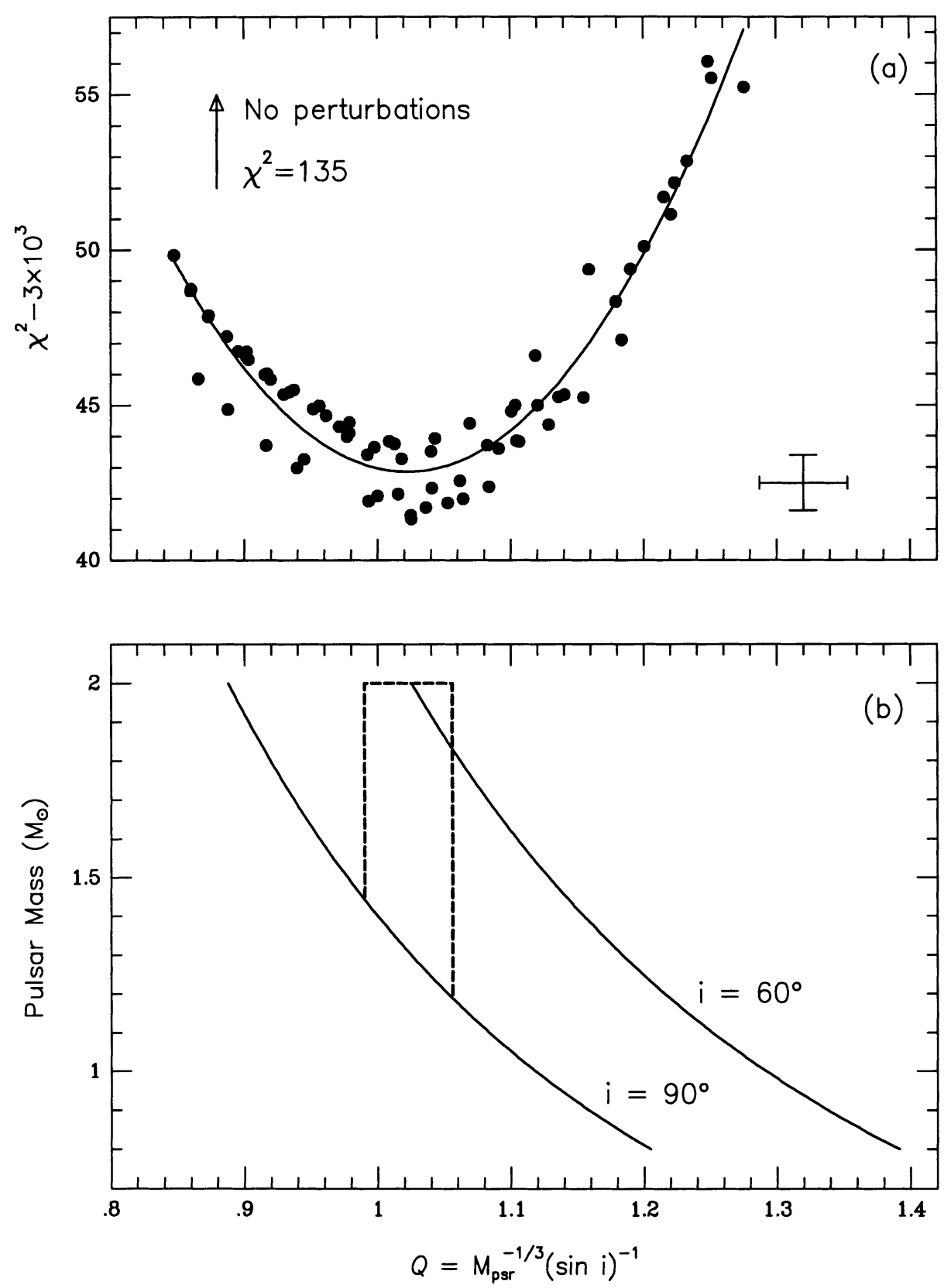

Figure 3. (a) A fit of the numerical models of the gravitational perturbations of planets $B$ and $C$ (filled circles) to pulse arrival times. The solid line represents the resultant best parabolic fit to the $\chi^{2}$ data. A $2 \sigma$ uncertainty of the minimum $\chi^{2}$ obtained from this fit is shown by the horizontal error bar. The vertical bar denotes the accuracy of the downhill simplex minimization procedure. (b) Constraints on the pulsar mass, the masses of planets $B$ and $C$ and the common orbital inclination. The dashed lines delimit the area containing the most likely combinations of these parameters. 


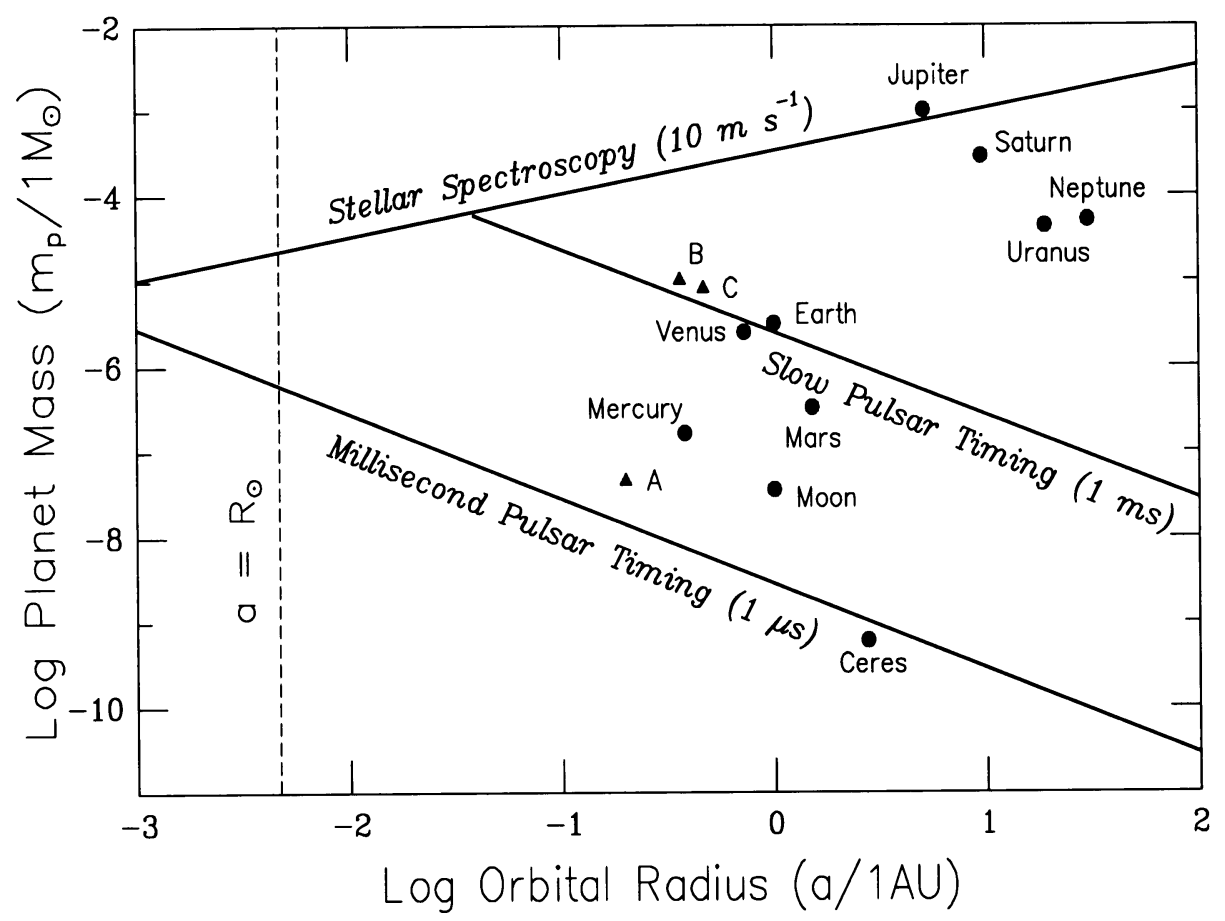

Figure 4. A comparison of the planet detection capabilities of the techniques of stellar spectroscopy and pulse timing. The assumed stellar and pulsar masses are $1 \mathrm{M}_{\odot}$ and $1.4 \mathrm{M}_{\odot}$, respectively. The filled circles and triangles mark the respective positions of the Solar System planets and the planets around PSR B1257+12 in the discovery space. The vertical dashed line sets the lower limit to orbital size defined by the solar radius.

five solitary objects that have apparently managed to dispose of their binary stellar companions. If a missing stellar companion to the pulsar indicates a possibility of "leftover" planets around it, PSR B1257+12 is the only confirmed case. Clearly, the pulsar planet formation is not $100 \%$ efficient, but the available statistics are too small to reliably constrain this efficiency. In addition, there is a large body of almost 600 younger, "slow" pulsars, some of which may have retained planets of their parent stars (Thorsett \& Dewey 1993). In fact, two of such pulsars have been reported to exhibit TOA variations that could be explained in terms of planetary dynamics (Demiański \& Prószyński 1979; Bailes et al. 1993; Lyne 1994). Extensive timing measurements and more direct observations of as many of these objects as possible will establish the significance of pulsar planetary systems as a class of astrophysical objects and their relationship to still hypothetical planets around Sun-like stars. 
Acknowledgements. I thank F. Camilo, R. Foster and A. Vázquez for help with observations, R. Malhotra and F. Rasio for discussions and S. Peale and J. Taylor for their contributions to the results presented in this paper. This research was supported by NASA under grant NAGW-3405 and by the NSF under grant AST-9317757. Arecibo Observatory is part of the National Astronomy and Ionosphere Center, which is operated by Cornell University under contract with the NSF.

\section{References}

Bailes, M., Lyne, A.G. \& Shemar, S.L. 1993, in Planets around Pulsars, J.A. Phillips, S.E. Thorsett \& S.R. Kulkarni (Eds.), ASP Conf. Ser. 36, 19

Bhattacharya, D. \& Van den Heuvel, E.P.J. 1991, Phys. Rep. 203, 1

Demiański, M. \& Prószyński, M. 1979, Nat 282, 383

Levy, E.H. 1993, in Planets around Pulsars, J.A. Phillips, S.E. Thorsett \& S.R. Kulkarni (Eds.), ASP Conf. Ser. 36, 181

Lyne, A.G. 1994, in Millisecond Pulsars: A Decade of Surprise, A.S. Fruchter, M. Tavani \& D.C. Backer (Eds.), ASP Conf. Ser. (in press)

Malhotra, R. 1993, ApJ 407, 266

Malhotra, R., Black, D., Eck, A. \& Jackson, A. 1992, Nat 356, 583

Nelder, J.A. \& Mead, R. 1965, Computer J. 7, 308

Peale, S.J. 1993, AJ 105, 1562

Phinney, E.S. \& Hansen, B.M.S. 1993, in Planets around Pulsars, J.A. Phillips, S.E. Thorsett \& S.R. Kulkarni (Eds.), ASP Conf. Ser. 36, 371

Podsiadlowski, P. 1993, in Planets around Pulsars, J.A. Phillips, S.E. Thorsett \& S.R. Kulkarni (Eds.), ASP Conf. Ser. 36, 149

Rasio, F.A. et al. 1992, Nat 355, 325

Rasio, F.A. et al. 1993, in Planets around Pulsars, J.A. Phillips, S.E. Thorsett \& S.R. Kulkarni (Eds.), ASP Conf. Ser. 36, 107

Ruden, S.P. 1993, in Planets around Pulsars, J.A. Phillips, S.E. Thorsett \& S.R. Kulkarni (Eds.), ASP Conf. Ser. 36, 197

Sargent, A.I. \& Beckwith, S.V.W. 1993, Phys. Today 46, 22

Thorsett, S.E. \& Dewey, R.J. 1993, ApJ 419, L65

Thorsett, S.E. et al. 1993, ApJ 405, L29

Wolszczan, A. 1991, Nat 350, 688

Wolszczan, A. 1994, Science 264, 538

Wolszczan, A. \& Frail, D.A. 1992, Nat 355, 145

Woosley, S.E. 1987, in The Origin and Evolution of Neutron Stars, IAU Symp. 125, D.J. Helfand \& J.H. Huang (Eds.), Reidel, p. 255 